\title{
A RARE UNUSUAL CASE OF SILICOSIDEROSIS MIMICKING SARCOIDOSIS
}

Daspin D', Abhin Joseph², Bhavik Patel ${ }^{3}$,Francis Gananprakasam 4

${ }_{1}^{1}$ MBBS, D.M.R.D. (D.N.B), Department of Radiodiagnosis, MIOT International Hospital, Chennai.

2MBBS, Department of Radiodiagnosis, MIOT International Hospital, Chennai.

3MBBS, Department of Radiodiagnosis, MIOT International Hospital, Chennai.

${ }^{4}$ MBBS, D.M.R.D. D.N.B., MNAMS, Department of Radiodiagnosis, MIOT International Hospital, Chennai.

\begin{tabular}{l}
\hline ABSTRACT \\
BACKGROUND \\
The current study reports the case of a 67-year-old gentleman who presented in our Institute with complaints of shortness of breath \\
and cough for last 8 months. He was working in a steel industry for almost 40 years. On evaluation further, he was diagnosed to have \\
occupational lung disease which was mimicking sarcoidosis and lymphoma.
\end{tabular}

\section{KEYWORDS}

Silicosiderosis, Tuberculosis, Sarcoidosis, Lymphoma, EBUS - Endobronchial Ultrasound and Biopsy, Anthracosis, AFB - Acid Fast Bacilli.

HOW TO CITE THIS ARTICLE: Daspin D, Joseph A, Patel B, et al. A rare unusual case of silicosiderosis mimicking sarcoidosis. J. Evolution Med. Dent. Sci. 2016;5(98):7222-7223, DOI: 10. 14260/jemds/2016/1634

\section{BACKGROUND}

A 67-year-old male, a retired plant worker from Jharkhand district came to our Institute with complaints of shortness of breath and cough for 8 months on evaluation. Initial evaluation with the chest $\mathrm{x}$-ray showed inhomogeneous opacities with nodular calcific foci in the right upper lobe. Bilateral hilar shadows also appear prominent.

Following this the contrast enhanced CT chest showed juxtapleural based spiculated soft tissue density mass with specks of calcification in the right upper lobe. Multiple calcified lymph nodes are also noted in the bilateral hilar region. Based on the imaging features the possibilities made were Sarcoidosis, TB and Lymphoma.

Other investigations that are done including culture showed absence of AFB cells. EBUS-guided transbronchial needle aspiration smears from region of right interlobar lymph node and subcarinal lymph node showed no lymphoid tissue. Cytology smears showed negative for malignant cells. At this point of time, no conclusive diagnosis was made from diagnostic and other investigations. Based on the CT findings ATT was started and after 2 months patient showed no improvement in clinical symptoms.

Repeat CT chest was done following which the soft tissue density lesion seen in the right upper lobe mass showed mild increase in size as compared to the previous scan and rightsided pleural effusion was also the new finding. Increase in the size of hilar lymph nodes was also noted.

Transbronchial lung biopsy was done showing ill-defined histiocytic aggregates, interstitial fibrosis and tiny foci of anthracosis. CT guided biopsy from the right upper lobe lesion was done under aseptic precautions. Biopsy showed granulomatous infiltrates and fibrosis with collagenisation, crystalline silica and hemosiderin deposits.

\footnotetext{
Financial or Other, Competing Interest: None.

Submission 26-10-2016, Peer Review 25-11-2016,

Acceptance 01-12-2016, Published 08-12-2016.

Corresponding Author:

Dr. Daspin D,

Department of Radiodiagnosis,

MIOT International Hospital,

Chennai-600089.

E-mail:daspins@yahoo.com

DOI: $10.14260 / \mathrm{jemds} / 2016 / 1634$

(c) $($ ) $\$$
}

Based on the correlation of the transbronchial findings and CT-guided lung biopsy findings, finally diagnosis of silicosiderosis with concomitant non-caseating granulomatous infiltrates was made.

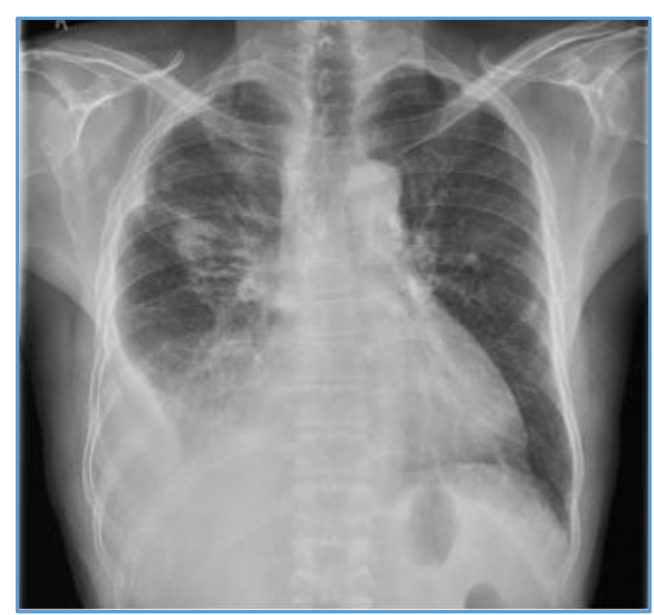

Figure 1a. Chest X-Ray showing Inhomogeneous Opacities with Nodular Calcific Foci in the Right Upper Lobe. Bilateral Hilar Shadows also appears Prominent with Minimal Right-Sided Pleural Effusion
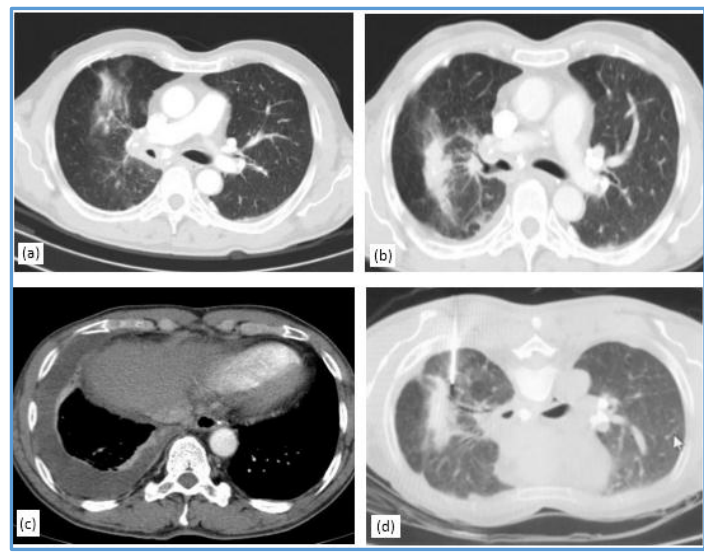

Figure 2a, b, c. Showing Juxtapleural Based Spiculated Soft Tissue Density Mass with Specks of Calcification in the Right Upper Lobe. Multiple Calcified Lymph Nodes are also Noted in the Bilateral Hilar Region. (d) Showing the Biopsy Images From Where the Samples are Obtained 


\section{DISCUSSION AND SUMMARY}

Silicosiderosis is caused by the accumulation of silica and iron oxide in macrophages within the lung. It is observed most commonly in workers exposed to metal fumes during welding and thus is known as arc welder pneumoconiosis (1\%). silicosiderosis is also found among tunnel highway construction workers who are digging through geological formations with large amounts of silica. In the 30 - 40s in the U.S., there was a huge epidemic of it in the Appalachians when they were building highways. The disease in its exacerbated form is far more debilitating and deadly than black lung disease. Silicosiderosis usually manifests with respiratory symptoms, such as cough and dyspnoea.(1,2)

In general, siderosis is not associated with fibrosis or functional impairment. However, when the iron inhaled is admixed with a substantial quantity of silica, the result is silicosiderosis which may be associated with pulmonary fibrosis. ${ }^{(3,4)}$

Typical radiographic findings include small nodules, which are most prominent in the middle-third of the lungs, in perihilar regions. The nodules do not indicate reactive fibrosis but rather radiopaque accumulations of iron particles in macrophages aggregated along perivascular and peribronchial lymphatic vessels.(5,6) In a thin-section CT-histopathologic study of 21 arc welders, Akira et al reported that CT scans showed small ill-defined centrilobular nodules $(71 \%$ of subjects) with or without fine branching structures indicating the deposition of minute iron oxide particles along perivascular and peribronchial lymphatic vessels; emphysematous changes $(33 \%$ of subjects $)$ and honeycombing resembling that in usual interstitial pneumonia $(14 \%) \cdot(6,7)$

Other findings included ground-glass opacities, reticulation, pleural irregularity and conglomerate masses with areas of high attenuation indicating organising pneumonia with siderosis. The transbronchial lung biopsy specimen obtained with visible light shows intra-alveolar aggregation of pigmented macrophages and scattered interstitial silica particles. The pathologic changes are reversible and complete resolution is possible after the cessation of exposure.(8) Patient improved symptomatically after treatment and is on regular followup for last 2 years. $(9,10)$

\section{CONCLUSION}

Silicosiderosis is listed as a "rare disease" by the Office of Rare Diseases (ORD) of the National Institutes of Health (NIH). Good clinical data and history of occupational exposure with radiological findings correlation will be able to more quickly and definitely render a diagnosis.

\section{REFERENCES}

1. Sherson D. Silicosis in the twenty first century. Occup Environ Med 2002;59(11):721-2.

2. Kuschner WG, Stark P. Occupational lung disease. Part 2. Discovering the cause of diffuse parenchymal lung disease. Postgrad Med 2003;113(4):81-8.

3. Onder M, Onder S. Evaluation of occupational exposures to respirable dust in underground coal mines. Ind Health 2009;47(1):43-9.

4. Ogawa S, Imai H, Ikeda M. Mortality due to silicotuberculosis and lung cancer among 200 whetstone cutters. Ind Health 2003;41(3):231-5.

5. Wagner GR. Asbestosis and silicosis. Lancet 1997;349(9061):1311-5.

6. Chong S, Lee KS, Chung MJ, et al. Pneumoconiosis: comparison of imaging and pathologic findings. Radiographics 2006;26(1):59-77.

7. Akira M. Uncommon pneumoconioses: CT and pathologic findings. Radiology 1995;197(2):403-9.

8. Fujimura N. Pathology and pathophysiology of pneumoconiosis. Curr Opin Pulm Med 2000;6(2):140-4.

9. Garg K, Lynch DA. Imaging of thoracic occupational and environmental malignancies. J Thorac Imaging 2002;17(3):198-210.

10. Franzblau A, Kazerooni EA, Sen A, et al. Comparison of digital radiographs with film radiographs for the classification of pneumoconiosis. Acad Radiol 2009;16(6):669-77. 